\title{
Neutrino cosmology
}

\section{Julien Lesgourgues*†}

LAPTH Annecy

E-mail: ']- 'esgourg@iapp.in2p3.fn'

ABSTRACT: I will briefly summarize the main effects of neutrinos in cosmology - in particular, on the Cosmic Microwave Background anisotropies and on the Large Scale Structure power spectrum. Then, I will present the constraints on neutrino parameters following from current experiments.

\section{A powerful tool: cosmological perturbations}

The theory of cosmological perturbations has been developed mainly in the 70's and 80's, in order to explain the clustering of matter observed in the Large Scale Structure (LSS) of the Universe, and to predict the anisotropy distribution in the Cosmic Microwave Background (CMB). This theory has been confronted with observations with great success, and accounts perfectly for the most recent CMB data (the WMAP satellite, see Spergel et al. 2003 [i]i and LSS observations (the 2dF redshift survey, see Percival et al. $2003[\overline{2} \overline{2}]$ ).

LSS data gives a measurement of the two-point correlation function of matter perturbations in our Universe, which is related to the linear power spectrum predicted by the theory of cosmological perturbations on scales between 40 Mega-parsecs (smaller scales correspond to non-linear perturbations, because they were enhanced by gravitational collapse) and 600 Mega-parsecs (larger scales are difficult to observe because galaxies are too faint). On should keep in mind that the reconstruction of the power spectrum starting from a redshift survey is complicated by many technical issues, like the correction of redshift-space distortions, and relies on strong assumptions concerning the light-to-mass biasing factor.

CMB data measures the Fourier spectrum of photon perturbations at the time of photon decoupling, at a redshift of approximately 1100 . This data is very complementary to LSS data because it probes the theory of cosmological perturbations at a much earlier time, and on a wider range of scales, from 20 Mega-parsecs to the current horizon scale (approximately 26000 Mega-parsecs). Compared to LSS, the spectrum of CMB anisotropies is a clean and robust observable, and leads to typically smaller error bars.

${ }^{*}$ Speaker.

${ }^{\dagger}$ This presentation follows various collaborations with Sergio Pastor. 
Let us summarize some striking feature of the theory of perturbations, in order to identify later the specific effect of neutrinos. Let us assume that we decompose all perturbations (including densities, pressures, phase-space distribution functions and metric fluctuations) in comobile Fourier space. Since comobile coordinates are related to physical distances through a scale factor $a(t)$, a perturbation of fixed comobile wavenumber has a time-dependent physical wavelength $\lambda(t)=2 \pi a(t) / k$. It is interesting to compare the growth of physical wavelengths with the physical scale set by general relativity, the Hubble radius $R_{H}(t)=a c d t / d a$. The Hubble radius plays the role of a causal horizon for any physical process starting after the end of inflation. It is straightforward to show that during radiation domination and matter domination, the Hubble radius grows more slowly than the scale factor. This proves that the cosmological scales which are observable today were outside of the Hubble scales at earlier time.

After their generation during inflation, cosmological perturbations undergo a long super-Hubble evolution during which none of the usual physical processes can occur. The scales smaller than approximately 300 Mega-parsecs entered inside the Hubble radius when the Universe was still radiation dominated. After Hubble crossing, the electromagnetic and gravitational interactions could operate causally, causing a propagation of density waves in the tightly coupled baryon-photon fluid. At the time of decoupling, which is soon after the equality between radiation and matter densities, all sub-Hubble scales carry an imprint of these coherent acoustic oscillations, in the form of peaks in the Fourier spectrum. Since photons free-stream after decoupling, this structure is conserved until today, and explains the famous series of acoustic peaks that we observe in the CMB anisotropy spectrum. So, we understand that the characteristic angular scale under which we see the first acoustic peak is related to the scale of the Hubble radius at decoupling - the scale of order 300 Mega-parsecs mentioned earlier.

But the perturbations of the non-relativistic fluids (dark matter, baryons) have a nontrivial evolution after the time of equality: during matter domination, gravitational collapse enhances any initial fluctuations and leads to the formation of the large scale structure of the Universe. The scales greater than approximately 300 Mega-parsecs do not have much time for enhancement, because they enter into the Hubble radius after equality. So, we understand that on this scale, there should be a break in the Fourier spectrum of matter perturbations.

So far, we did not mention the role of neutrinos. In the current version of the standard cosmological model, five fluids contribute significantly to the density of the Universe: photons, baryons, cold dark matter (CDM), neutrinos and a cosmological constant. After neutrino decoupling, the neutrinos are essentially collisionless, like CDM, and unlike photons and baryons. So, they interact only gravitationally with the other species, which means that their influence on the evolution of the cosmological perturbations is entirely described by the Einstein equation. Usually, the latter is decomposed into some background equations (mainly, the Friedmann equation, relating the background densities to the time-derivative of the scale factor) and a complicated set of perturbed equations (which generalize the usual Poisson equation of Newtonian gravity). At the background level, the neutrinos appear in the Friedmann equation; by changing the expansion rate of the Uni- 
verse, they can modify some characteristic times and scales. At the perturbed level, the could either enhance or smooth metric perturbations, which would back-react on the evolution of matter perturbations. In the next sections, we will summarize the role first of massless neutrinos, and then of massive neutrinos. In each case, we will separate the total effect into a background and a perturbation effect.

\section{Massless neutrinos}

Today, we can directly measure the density of photons (through the CMB temperature), of a cosmological term (through the luminosity distance-redshift relation), and of CDM plus baryons (through the curvature of the Universe and the Hubble parameter). For the neutrino background, one can think of indirect probes, but not of a direct measurement. However, a neutrino background has been assumed since a long time, because we follow a theoretical prejudice, based on the standard model of neutrino decoupling: the early Universe would contain the three flavor neutrinos at thermal equilibrium with the photon background; then, after decoupling, the ratio of the neutrino density $\rho_{\nu}$ and the photon density $\rho_{\gamma}$ would remain constant. In the "instantaneous decoupling" picture, this ratio is given by the number of families $N_{\nu}=3$, by the factor $7 / 8$ accounting for the number of degrees of freedom of fermions relative to that of bosons, and finally by a factor $(4 / 11)^{1 / 3}$ representing the enhancement of the neutrino temperature relatively to that of photons during neutrino decoupling.

This relation is a theoretical prejudice, that needs to be tested with observations. For this purpose, the density $\rho_{\nu}$ can be considered as a free parameter that we want to measure. Alternatively, we can decide to employ the previous formula, but now considering $N_{\nu}$ as a free parameter, bigger or smaller than three. This convention is the most usual one, and $N_{\nu}$ is then called the " effective neutrino number", that should be clearly distinguished from the actual number of flavor species, know to be equal to three from accelerator experiments. Measuring $N_{\nu}$ is not a way of corroborating the accelerator results, but rather of testing various assumptions concerning the density of relativistic particles in our Universe. More precisely, the measurement of $N_{\nu}$ is interesting for at least four reasons:

1. to test the presence of extra relativistic particles in the Universe, beyond the three standard neutrinos (some exotic candidates are sterile neutrinos, axions, light gravitinos, majorons, an effect of extra spatial dimensions, etc.) These would presumably increase $N_{\nu}$ beyond three.

2. to test the standard model of neutrino decoupling. By pure convention, the definition of $N_{\nu}$ is based on the above-mentioned formula, describing neutrino decoupling in the so-called "instantaneous approximation". But in more realistic models, neutrinos are not fully decoupled at the time of electron-positron annihilation, and get slightly reheated; in addition, finite temperature QED corrections produce a slight distortion of the neutrino phase-space distribution function with respect to a pure Fermi-Dirac expression. The net effect can be summarized by an effective neutrino number equal to 3.04 instead of 3 [ij]. This number should be taken as the standard prediction. 
However, one can build some models of neutrino decoupling that will be, for some reason, out of thermal equilibrium. This can result in an effective neutrino number considerably bigger or smaller than 3.04 .

3. to test Big Bang Nucleosynthesis. Changing the effective neutrino number leads to a different expansion rate at the time of Nucleosynthesis, and therefore, to different relic abundances of light elements. The observed abundances point to $\left|N_{\nu}-1\right| \leq 1$, assuming standard nucleosynthesis [i] (inhomogeneous, degenerate, etc.) are still compatible with a much higher number. Therefore, measuring $N_{\nu}$ can be a way of discriminating between various alternatives to standard nucleosynthesis - though, keeping in mind that when $N_{\nu}$ is measured through cosmological perturbations, what is really probed is the number of relativistic degrees of freedom at redshifts between 100 and 10000, while nucleosynthsis is sensitive to this number at much higher redshift. In some exotic scenarios, the number of relativistic degrees of freedom can change after nucleosynthesis due to the decay of massive particles, so one should be careful before drawing any robust conclusion [יi5].

4. to test the neutrino-antineutrino asymmetry. The number density of neutrinos and anti-neutrinos is generally assumed to be the same. This is not necessarily true in some models, and the asymmetry might lead to an interesting phenomenology "6 6 i However, the discovery of neutrino flavor oscillations has put a strict upper bound on the asymmetry parameter, using again nucleosynthesis [i]]: so, we expect that an eventual asymmetry could not increase $N_{\nu}$ by more than $10^{-5}$. However, a direct measurement of the asymmetry is still interesting.

We explained why it is exciting to measure $N_{\nu}$ with a method based on cosmological perturbations. We will say now how it can be done.

The first effect of a variation of $N_{\nu}$ is a background effect. Since the current density of non-relativistic matter (baryons and cold dark matter) is known, changing the radiation content tunes the time of equality between matter and radiation. On the other hand, the time of decoupling is more or less fixed. So, the parameter $N_{\nu}$ gives the relative amount of radiation at the time of decoupling. This has a direct consequence on the amplitude of the first CMB acoustic peaks, through the so-called "integrated Sachs-Wolfe effect": more radiation enhances the first peak. Also, the sound horizon at decoupling (which governs the exact wavelength of the acoustic peaks) depends on $N_{\nu}$. This is easy to understand: the horizon is defined as the distance traveled by light since an initial time. By changing the time of equality, one changes the Universe expansion before decoupling: so, the sound horizon at decoupling is altered. Concretely, an increase in $N_{\nu}$ shifts the acoustic peaks to smaller wavelengths. Finally, still at the background level, there is an effect of $N_{\nu}$ over the matter power spectrum (describing LSS). It should be clear from the discussion in the first section that the time of equality defines the scale of the turn-over in this spectrum. So, increasing $N_{\nu}$ shifts this turn-over to larger scales. Also, since equality takes place later, 
there is less time for the growth of matter perturbations during matter domination. So, and increase in $N_{\nu}$ also suppresses power at small wavelength.

We just described the effect of varying $N_{\nu}$ on the CMB and LSS spectra, at the level of background equations. There is also an effect at the level of perturbed equations, but it is more difficult to explain, and affects the spectra more or less in the same way. We will not enter into these details here.

So, it is clear that a precise measurement of the CMB anisotropy and LSS power spectra provides a chance to measure the abundance of relativistic relics in our Universe. The main limitation to this method comes from the fact that the cosmological power spectra are sensitive to a number of cosmological parameters, whose individual effects cannot be easily disentangled. Therefore, the error bar for one parameter heavily depends on the assumptions made concerning the other parameters. In particular, the effect of $N_{\nu}$ is almost degenerate with that of the matter density, because if the two parameters are varied simultaneously in such way that the time of equality is kept fixed, the power spectra do not vary much. Therefore, most authors use a prior on the value of the Hubble parameter (coming from the Hubble Space Telescope Key Project determination) and of the cosmological constant (from the Supernovae Cosmology Project) which fixes the matter density (given that the CMB spectrum indicates a nearly flat Universe).

Taking the most recent data (one-year observations by WMAP, and the matter power spectrum reconstructed by the $2 \mathrm{dF}$ survey), Ref. [8.] gets $-1.6<\left(N_{\nu}-3\right)<3.8$ at the $95 \%$ confidence level, assuming an exactly flat Universe. Relaxing the previous assumptions, Ref. [īin] gets similar bounds.

In the future, it is expected that the Planck satellite CMB observations, combined with the final matter power spectrum obtained from the Sloan Digital Sky Survey (SDSS), will allow for a measurement of $N_{\nu}$ with a precision of 0.3 (at the one-sigma level) [i $\left.1 \overline{1}_{1}^{1}\right]$. Unfortunately, this is not sufficient for testing the difference between $N_{\nu}=3$ and the predictions of the non-instantaneous neutrino decoupling model. However, it is remarkable that the final precision of the CMB+LSS measurement will be comparable to that of Nucleosynthesis, allowing for a very interesting confrontation.

\section{Massive neutrinos}

So far, we assumed that the neutrino species (three or more) were massless. This is a reasonable first-order approximation: as we shall see, the neutrino masses are bounded to be small (smaller than $1 \mathrm{eV}$ ), and the cosmological effect of the mass is tiny. Therefore, the bound that we presented previously on the number of massless neutrinos also apply to sub-eV massive neutrinos. However, in this section, we will focus on the specific effect of the mass on the evolution of cosmological perturbations and on its detectability.

In the early universe, massive neutrinos are relativistic and indistinguishable from massless ones. Their energy density drops like $a^{-4}$, like that of photons. When their average momentum (which gets redshift with the Universe expansion, proportionally to $a^{-1}$ ) drops below the mass of a given eigenstate, the corresponding neutrinos become nonrelativistic, and their energy density evolves like that of baryons and cold dark matter 
(like $a^{-3}$ ). For $\mathrm{eV}$ or sub-eV masses, the relativistic to non-relativistic transition occurs after the time of equality between photons+neutrinos and baryons+CDM. Therefore, the difference between the massless and massive scenarios is visible only at late time; then, the density of neutrinos tends to a fixed fraction $\Omega_{\nu}$ of the critical density of the Universe (actually, $\Omega_{\nu}$ ies not exactly constant at late times because of the Universe acceleration). If all neutrino species are massive, the value of $\Omega_{\nu}$ depends linearly on the sum $\sum_{i} m_{i}$, where $i$ labels each mass eigenstate. Cosmology is completely blind to the fact that neutrinos are Dirac or Majorana and to the mixing angles. The coefficient of proportionality is approximately equal to $\Omega_{\nu}=\sum_{i} m_{i} /(50 \mathrm{eV})$ : so, neutrino with $\sum_{i} m_{i}=0.5 \mathrm{eV}$ contribute to $1 \%$ of the total energy density of the Universe today.

The effect of light neutrinos on the CMB is small and indirect, because at the time of decoupling, neutrinos are still relativistic (although they might be starting their transition). However, the main parameter that we can extract from the CMB spectrum are the current densities of the cosmological constant, of baryons, of cold dark matter and of neutrinos. If one varies the latter, keeping the first three fixed, one changes the total energy density, and therefore, the Universe curvature; this change in the geometry will shift the angular scale under which we see the peaks. Alternatively, one might decide to fix the Universe curvature to zero, obeying to a theoretical prior; then, a change in $\Omega_{\nu}$ must be consistently compensated by a change in one of the three other components. This will inevitably affect at least the time of equality between matter and radiation, with an effect on the CMB comparable to the one of $N_{\nu}$.

The effect of light neutrinos on the LSS power spectrum is two-fold. At the background level, the situation is the same as for the CMB: the change in $\Omega_{\nu}$ has consequences on the time of equality. But the dominant effect arises at the level of perturbations, and is called the free-streaming effect. Even when they become non-relativistic, neutrinos have a velocity. So, on small scales, they travel over small potential wells without feeling them, and tend to smooth the inhomogeneities; on large scales, they get confined inside the largest potential wells. The smoothing of small-scale perturbations is efficient below the so-called Jeans length of the photons; when they are still in the relativistic regime, this Jeans length matches the causal horizon $R_{H}$, while afterward, it is bounded by $(<p>/ m) R_{H}$, where $\langle p\rangle$ is the average momentum, decreasing like $a^{-1}$. The net effect of the mass on the LSS power spectrum is a suppression which is maximal on wavelengths smaller than the present value of the neutrino Jeans length (also called the free-streaming scale).

The free-streaming effect is the dominant one for massive neutrinos, and represent our best hope to measure or to bound from above the absolute mass scale of neutrinos. However, it is crucial to combine the LSS and the CMB power spectra: even if the latter is less sensitive to the mass, it plays a crucial role in removing parameter degeneracies. The main parameter to which this method is sensitive is the sum over the masses, $\sum_{i} m_{i}$. However, the CMB and LSS power spectra exhibit a small sensitivity to the way in which the total mass is split between the various eigenstates. Indeed, if we fix respectively $N_{\nu}$ and $\sum_{i} m_{i}$, we fix the Universe composition and expansion rate respectively in the early and late Universe. However, by choosing different splittings of $\sum_{i} m_{i}$ between the eigenstates, we still can change a little bit the time of the relativistic to non-relativistic regime: for 
instance, three neutrinos of mass $m_{1}$ become non-relativistic later than one neutrino of mass $3 m_{1}$ that would coexist with two nearly massless ones. This will produce a tiny difference in the CMB spectrum. Significantly bigger will be the difference in the free-streaming effect on matter perturbations, which depends on the individual mass of each species: on intermediate scales, three neutrinos of mass $m_{1}$ smooth the inhomogeneities more efficiently than one of mass $3 m_{1}$, with a larger free-streaming wavelength today. These differences exist in theory, but it is not clear that future experiments will reach enough sensitivity for extracting more parameters than just $N_{\nu}$ and $\sum_{i} m_{i}$.

Let us see how current experiments can bound realistic neutrino mass scenarios. Assuming three neutrino mass eigenstates, results from oscillations experiments privilege two mass schemes: a normal hierarchical scenario and an inverted hierarchical scenario; in these scenarios, the squared mass differences are fixed, but the largest mass is a free parameter, bounded only from below. Therefore, by increasing this largest mass, the two hierarchical scenarios can be extrapolated to a degenerate scenario, in which each of the squared masses is much bigger than the two differences $\left(\Delta m^{2}\right)$. From the point of view of cosmology, which is not sensitive to masses smaller than $0.1 \mathrm{eV}$, these three cases can be approximated by three schemes with respectively one, two or three massive degenerate neutrinos, and two, one or zero massless ones.

The sensitivity of current experiments is appropriate for putting a higher bound only on the last case, with three degenerate neutrinos. The result of combining WMAP and $2 \mathrm{dF}$ differs between various authors, who employ different priors on the cosmological parameters, and different techniques of parameter extraction. They vary between $\sum_{i} m_{i}<0.69$

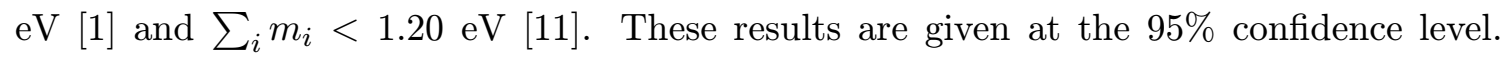
However, one should keep in mind that the statistical modeling of cosmological data is far from perfect: so, one should consider these error bars as a rough indication, rather than an exact $2-\sigma$ level.

These results hold for three-neutrino mass schemes. If the LSND result was confirmed by the MiniBoome experiment, it would be unavoidable to include at least one sterile neutrino. However, changing $N_{\nu}$ from 3 to 4 has a large effect on the CMB and LSS spectra, and in that case, the bound on $\sum_{i} m_{i}$ is different. ¡Ref. [i] i, in gives $\sum_{i} m_{i}<1.4 \mathrm{eV}$ for $N_{\nu}=4$. This bound is not restrictive enough for ruling out the LSND observation.

Let us conclude with a few words on the expected sensitivity of future experiments. The authors of [i1 $\left.{ }_{1}^{1} \overline{2}\right]$ found that combining Planck and SDSS would constrain the sum $\sum_{i} m_{i}$ with a one-sigma error of $0.3 \mathrm{eV}$. The author of [1] be marginally sufficient for discriminating between the hierarchical and inverse hierarchical scenarios, but this result assumes an ideal CMB experiment, limited only by cosmic variance (i.e., by the "theoretical uncertainty" on the CMB power spectrum).

\section{References}

[1] D. N. Spergel et al., Astrophys. J. Suppl. 148 (2003) 175 [arXiv:astro-ph/0302209].

[2] W. J. Percival et al., Mon. Not. Roy. Astron. Soc. 327 (2001) 1297 [arXiv:astro-ph/0105252]. 
[3] G. Mangano, G. Miele, S. Pastor and M. Peloso, Phys. Lett. B 534 (2002) 8 [arXiv:astro-ph/0111408].

[4] A. Cuoco, F. Iocco, G. Mangano, G. Miele, O. Pisanti and P. D. Serpico, arXiv:astro-ph/0307213;

R. H. Cyburt, B. D. Fields and K. A. Olive, Phys. Lett. B 567 (2003) 227 [arXiv:astro-ph/0302431].

[5] M. J. White, G. Gelmini and J. Silk, Phys. Rev. D 51 (1995) 2669 [arXiv:astro-ph/9411098]; M. Kaplinghat and M. S. Turner, Phys. Rev. Lett. 86 (2001) 385 [arXiv:astro-ph/0007454].

[6] H. S. Kang and G. Steigman, Nucl. Phys. B 372 (1992) 494;

G. B. Larsen and J. Madsen, Phys. Rev. D 52 (1995) 4282;

J. A. Adams \& S. Sarkar, Oxford University preprint OUTP-98-70P and talk presented at the worshop on The Physics of Relic Neutrinos, Trieste, September 1998;

J. Lesgourgues and S. Pastor, Phys. Rev. D 60 (1999) 103521 [arXiv:hep-ph/9904411];

W. H. Kinney and A. Riotto, Phys. Rev. Lett. 83 (1999) 3366 [arXiv:hep-ph/9903459];

J. Lesgourgues and A. R. Liddle, Mon. Not. Roy. Astron. Soc. 327 (2001) 1307 [arXiv:astro-ph/0105361];

S. H. Hansen, G. Mangano, A. Melchiorri, G. Miele and O. Pisanti, Phys. Rev. D 65 (2002) 023511 [arXiv:astro-ph/0105385].

[7] A. D. Dolgov, S. H. Hansen, S. Pastor, S. T. Petcov, G. G. Raffelt and D. V. Semikoz, Nucl. Phys. B 632 (2002) 363 [arXiv:hep-ph/0201287].

[8] P. Crotty, J. Lesgourgues and S. Pastor, Phys. Rev. D 67 (2003) 123005 [arXiv:astro-ph/0302337].

[9] E. Pierpaoli, Mon. Not. Roy. Astron. Soc. 342 (2003) L63 [arXiv:astro-ph/0302465].

[10] R. E. Lopez, S. Dodelson, A. Heckler and M. S. Turner, Phys. Rev. Lett. 82 (1999) 3952 [arXiv:astro-ph/9803095];

J. Lesgourgues, S. Pastor and S. Prunet, Phys. Rev. D 62 (2000) 023001

[arXiv:hep-ph/9912363];

R. Bowen, S. H. Hansen, A. Melchiorri, J. Silk and R. Trotta, Mon. Not. Roy. Astron. Soc. 334 (2002) 760 [arXiv:astro-ph/0110636].

[11] S. Hannestad, JCAP 0305 (2003) 004 [arXiv:astro-ph/0303076].

[12] W. Hu, D. J. Eisenstein and M. Tegmark, Phys. Rev. Lett. 80 (1998) 5255 [arXiv:astro-ph/9712057].

[13] S. Hannestad, Phys. Rev. D 67 (2003) 085017 [arXiv:astro-ph/0211106]. 